\title{
On gravitational and thermal corrections to vacuum decay
}

\author{
Alberto Salvio, ${ }^{a}$ Alessandro Strumia, ${ }^{a, b}$ Nikolaos Tetradis ${ }^{a, c}$ and Alfredo Urbano ${ }^{a}$ \\ ${ }^{a}$ CERN, Theoretical Physics Department, \\ Geneva, Switzerland \\ ${ }^{b}$ Dipartimento di Fisica dell'Università di Pisa and INFN - Sezione di Pisa, \\ Largo Bruno Pontecorvo 3, Pisa, Italy \\ ${ }^{c}$ Department of Physics, University of Athens, \\ Zographou 15784 , Greece \\ E-mail: alberto.salvio@cern.ch, astrumia@cern.ch, ntetrad@phys.uoa.gr, \\ alfredo.leonardo.urbano@cern.ch
}

ABSTRACT: We reconsider gravitational corrections to vacuum decay, confirming and simplifying earlier results and extending them allowing for a non-minimal coupling of the Higgs to gravity, finding that leading-order gravitational corrections suppress the vacuum decay rate. Furthermore, we find minor corrections to thermal vacuum decay in the SM adding one-loop corrections to the Higgs kinetic term, two-loop corrections to the Higgs potential and allowing for time-dependent bounces.

Keywords: Higgs Physics, Thermal Field Theory, Quark Masses and SM Parameters, Renormalization Group

ARXiv EPrint: 1608.02555 


\section{Contents}

1 Introduction 1

2 Gravitational corrections to SM vacuum decay 1

2.1 The low-energy approximation 2

2.2 Gravitational corrections in a toy model 3

2.3 Gravitational corrections to Higgs vacuum decay 4

2.4 Effects of new Planckian physics 8

3 SM vacuum decay at finite temperature $\quad 9$

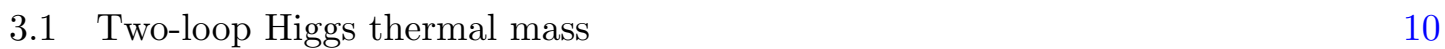

3.2 One-loop thermal correction to the Higgs kinetic energy 11

$\begin{array}{ll}3.3 & \text { Is the thermal bounce time-independent? } \\ \end{array}$

4 Conclusions $\quad 15$

\section{Introduction}

The measured Higgs mass lies close to the critical value above which the Standard Model (SM) Higgs potential is unstable at large field values. In order to determine if the SM predicts that our universe is stable or unstable, several precision calculations were performed recently [1-3], along with studies of gravitational corrections to vacuum decay [4-11] and of cosmological implications [12-23]. Also, the vacuum-decay formalism has been scrutinized [24], and better measurements of the top mass (the most unknown relevant parameter) are being planned, from electroweak data, flavour data, LHC data, and possibly new colliders [25]. We contribute to this effort by addressing two concrete issues.

Concerning the vacuum decay rate, we show in section 2 that analytical techniques for including gravitational corrections at leading order in the inverse Planck mass [4] provide correct results, contrary to the criticism of two recent papers $[7,10]$. We extend and simplify the results of [4].

Concerning the thermal tunnelling rate in the early universe, in section 3 we extend previous calculations that included the one-loop thermal potential [26-29], by adding oneloop thermal kinetic terms (section 3.2), two-loop thermal masses (section 3.1) and allowing for time-dependent bounces (section 3.3).

In section 4 we present our conclusions.

\section{Gravitational corrections to SM vacuum decay}

Coleman and De Luccia developed a formalism for studying vacuum decay taking gravity into account [30]. However, the full theory of quantum gravity is unknown: gravity is only 
known at the leading order in a low-energy expansion in inverse powers of $M_{\mathrm{Pl}}$. Thereby, the authors of [4] proposed a simple semi-analytic approximation that captures the leading gravitational correction to vacuum decay. The authors of $[7,10]$ performed brute-force numerical computations of gravitational corrections in Einstein gravity, and claim that the result of [4] is not valid. We show that the original result in [4] is correct by providing further details on how it is obtained; we simplify the analytic expressions of [4] and validate them through correct numerical computations. We also generalize [4] to the case of a nonminimal coupling between the Higgs and gravity.

\subsection{The low-energy approximation}

We consider the Euclidean Einstein-Hilbert-Higgs action

$$
S=\int d^{4} x \sqrt{g}\left[\frac{\left(\partial_{\mu} h\right)\left(\partial^{\mu} h\right)}{2}+V(h)-\frac{\mathcal{R}}{2 \kappa}-\frac{\mathcal{R}}{2} f(h)\right],
$$

where $\mathcal{R}$ is the Ricci scalar, $\kappa=1 / \bar{M}_{\mathrm{Pl}}^{2}=8 \pi G$ with $\bar{M}_{\mathrm{Pl}}=M_{\mathrm{Pl}} / \sqrt{8 \pi}, M_{\mathrm{Pl}} \approx 1.22 \times$ $10^{19} \mathrm{GeV}$. For the moment we assume that the potential $V(h)$ and $f(h)$ are generic functions of the scalar field $h(x)$. We allow for a generic non-minimal coupling to gravity $f(h)$, extending the formalism of $[4,30]$. We introduce an $\mathrm{O}(4)$-symmetric Euclidean ansatz for the bounce $h(r)$ and for its geometry

$$
d s^{2}=d r^{2}+\rho(r)^{2} d \Omega^{2},
$$

where $d \Omega$ is the volume element of the unit 3-sphere. On this background $S$ becomes

$$
S=2 \pi^{2} \int d r \rho^{3}\left[\left(\frac{h^{\prime 2}}{2}+V\right)-\frac{\mathcal{R}}{2 \kappa}-\frac{\mathcal{R}}{2} f(h)\right],
$$

where now $\mathcal{R}=-6\left(\rho^{2} \rho^{\prime \prime}+\rho \rho^{2}-\rho\right) / \rho^{3}$ and a prime denotes $d / d r$. The equations of motion are

$$
h^{\prime \prime}+3 \frac{\rho^{\prime}}{\rho} h^{\prime}=\frac{d V}{d h}-\frac{1}{2} \frac{d f}{d h} \mathcal{R}, \quad \rho^{\prime 2}=1+\frac{\kappa \rho^{2}}{3(1+\kappa f(h))}\left(\frac{h^{\prime 2}}{2}-V-3 \frac{\rho^{\prime}}{\rho} \frac{d f}{d h} h^{\prime}\right),
$$

where the latter equation can be obtained from the $r r$ component of the Einstein equations. The bounce action in eq. (2.3) can be simplified using a scaling argument analogous to that of [31]: the bounce action is stationary under the rescaling $g_{\mu \nu} \rightarrow s^{2} g_{\mu \nu}$. When this rescaling is implemented in eq. (2.1), evaluated for the solution of the equations of motion, the action should have an extremum at $s=1$. This observation relates the different contributions to the total integral that get multiplied by different powers of $s$. In particular, it implies that the bounce action can be simplified to

$$
S=-2 \pi^{2} \int d r \rho^{3} V
$$

evaluated on the solution of eq.s (2.4) with the boundary conditions appropriate for a bounce. This can only be solved numerically. 
Following [4] we include analytically the effect of gravity, assuming $R M_{\mathrm{Pl}} \gg 1$, where $R$ is the size of the bounce, by performing a leading order expansion in the gravitational coupling $\kappa$ :

$$
h(r)=h_{0}(r)+\kappa h_{1}(r)+\mathcal{O}\left(\kappa^{2}\right), \quad \rho(r)=r+\kappa \rho_{1}(r)+\mathcal{O}\left(\kappa^{2}\right) .
$$

The action $S_{0}$ at the 0th order in $\kappa$ is simply the scalar action in the absence of gravity computed for $h=h_{0} \cdot{ }^{1}$ The action expanded at leading order in $\kappa$ is

$$
S=S_{0}+\Delta S_{\text {gravity }}
$$

with

$$
\begin{aligned}
\Delta S_{\text {gravity }}=\frac{6 \pi^{2}}{\bar{M}_{\mathrm{Pl}}^{2}} \int d r[ & r^{2} \rho_{1}\left(\frac{h_{0}^{\prime 2}}{2}+V\left(h_{0}\right)\right) \\
& \left.+\left(r \rho_{1}^{\prime 2}+2 \rho_{1} \rho_{1}^{\prime}+2 \rho_{1} r \rho_{1}^{\prime \prime}\right)+r f\left(h_{0}\right)\left(r \rho_{1}^{\prime \prime}+2 \rho_{1}^{\prime}\right)\right] .
\end{aligned}
$$

Notice that $h_{1}$ does not appear in eq. (2.8). The general reason behind this is that the Higgs field sources gravity, but gravity does not source the Higgs. A simplification of the above expression is possible through arguments similar to the one that led to eq. (2.5). The total action can be viewed as a functional of $\rho(r)$ and $h(r)$, minimized for the solution of eq.s (2.4). Rescaling $\rho_{1}(r) \rightarrow s \rho_{1}(r)$ corresponds to shifting the solution of the equations of motion by $(s-1) \rho_{1}(r)$ (notice that this variation vanishes at the endpoints). The action must have an extremum at $s=1$. Applying this argument to (2.8), by rescaling $\rho_{1}(r) \rightarrow s \rho_{1}(r)$ and requiring that the $s$-derivative of the resulting expression vanishes at $s=1$, relates the integrals of terms linear and quadratic in $\rho_{1}$. It leads to

$$
\Delta S_{\text {gravity }}=-\frac{6 \pi^{2}}{\bar{M}_{\mathrm{Pl}}^{2}} \int d r\left(r \rho_{1}^{\prime 2}+2 \rho_{1} \rho_{1}^{\prime}+2 \rho_{1} r \rho_{1}^{\prime \prime}\right)=\frac{6 \pi^{2}}{\bar{M}_{\mathrm{Pl}}^{2}} \int d r r \rho_{1}^{\prime 2} \geq 0
$$

where the last equation is obtained trough an integration by parts and is manifestly positive. Once $h_{0}$ is known, $\rho_{1}^{\prime}$ is given by eq. (2.4) expanded at leading order in $\kappa$ :

$$
\rho_{1}^{\prime}=\frac{r^{2}}{6}\left[\frac{h_{0}^{\prime 2}}{2}-V\left(h_{0}\right)-\frac{3}{r} f^{\prime}\left(h_{0}\right) h_{0}^{\prime}\right]
$$

where $f^{\prime}\left(h_{0}\right)$ is the derivative of $f$ with respect to $h$ evaluated at $h=h_{0}$. Inserting this expression in eq. (2.9) gives the leading order gravitational correction to the action. Only an integration is needed.

\subsection{Gravitational corrections in a toy model}

Branchina et al. [7] performed a numerical analysis of vacuum decay that resulted in the claim: "the output of [4] cannot be trusted and a fortiori cannot be used for comparison".

\footnotetext{
${ }^{1}$ The action contains the curvature term enhanced by negative powers of the Planck mass. Its expansion $-\sqrt{g} \mathcal{R} /(2 \kappa)=3\left(r^{2} \rho_{1}^{\prime}\right)^{\prime}+\mathcal{O}(\kappa)$ apparently produces an extra 0th-order term. However, this total-derivative term gives no contribution to $S_{0}$ for a $\rho_{1}^{\prime}$ that is regular in $r=0$ and falls off sufficiently fast as $r \rightarrow \infty$.
} 

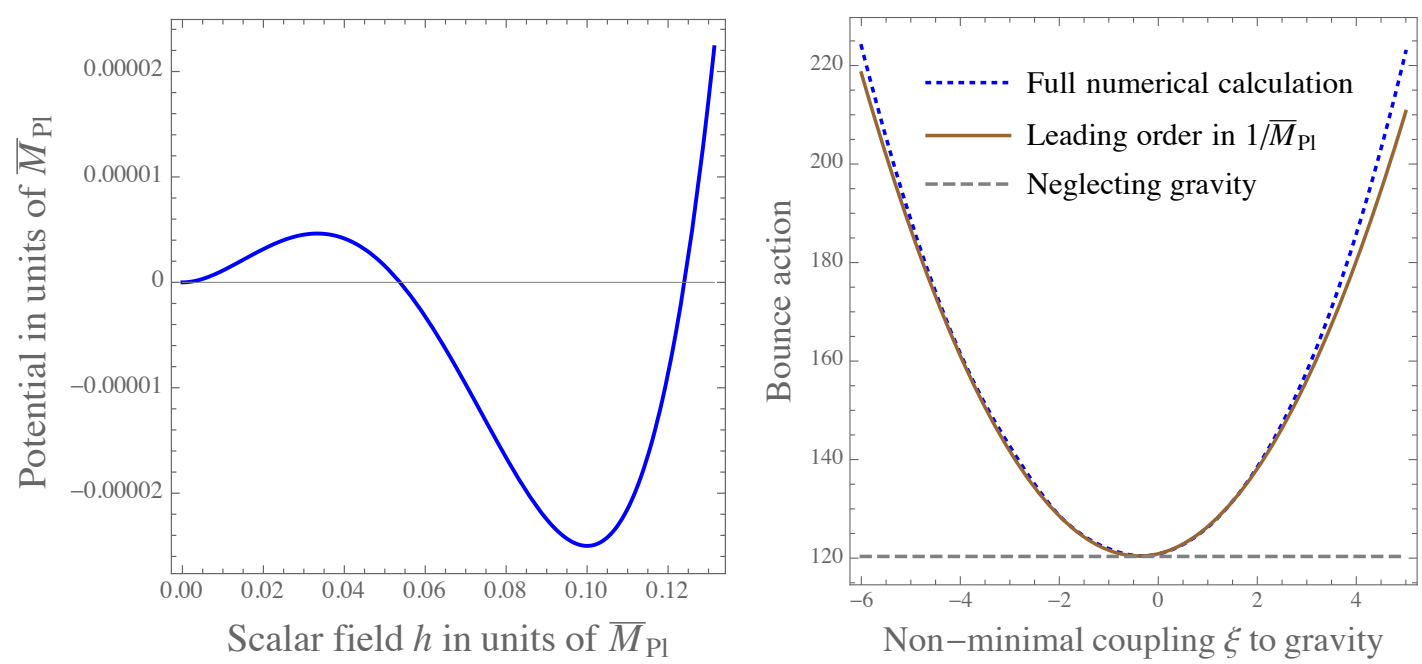

Figure 1. Left: the potential (2.11) for $g=3, b=1 / 3$ and $a=0.05 \bar{M}_{\mathrm{Pl}}$. Right: the corresponding bounce action as a function of the non-minimal coupling $\xi$, comparing the full numerical result with the approximation at leading order in $1 / M_{\mathrm{Pl}}$.

We perform the comparison between numerical results and the semi-analytical approximation of [4] for gravitational corrections to vacuum decay, and find perfect agreement. We consider the same quartic scalar potential studied in $[7]^{2}$

$$
V(h)=\frac{g^{2}}{4}\left\{\left[(h-a)^{2}-a^{2}\right]^{2}+\frac{4 b}{3}\left[a(h-a)^{3}-3 a^{3}(h-a)-2 a^{4}\right]\right\}-V_{0} .
$$

The left panel of figure 1 shows the potential for a sub-Planckian choice of its parameters $g$, $a$ and $b$, and $f(h)=\xi h^{2}$. In the right panel we show the bounce action as a function of $\xi$ in three cases: i) ignoring gravity; ii) including gravity, with the perturbative approximation of eq. (2.9); iii) including gravity, performing a full numerical computation of eq. (2.5).

We see that the perturbative approximation reproduces the full numerical result. For $\xi=0$ (the value considered in [7]) and the input values considered in figure 1 , we find $S_{0} \approx 120.3$ and $S \approx 120.9$, which agrees with the perturbative approximation at the permille level. For larger values of $\xi$ gravity becomes stronger, and the perturbative expansion starts to break down, as expected. We emphasize that a full numerical computation does not lead in an increase in precision, because the semi-classical approximation too breaks down when gravity becomes strong unknown quantum-gravity effects generically become relevant, as discussed in section 2.4.

\subsection{Gravitational corrections to Higgs vacuum decay}

Rajantie and Stopyra [10] reconsidered the gravitational corrections to the vacuum decay rate in the Standard Model, concluding that: "our numerical results are in conflict with [4]". We perform one more numerical computation, finding agreement with the analytic results of [4] and clarifying the issues that led to the misunderstanding in [10].

\footnotetext{
${ }^{2}$ With respect to the conventions of [7], we have shifted the field so that the local minimum is located at $h=0$, and added a constant $V_{0}$ to the potential so that $V_{\mathrm{B}}(0)=0$ at the false vacuum.
} 


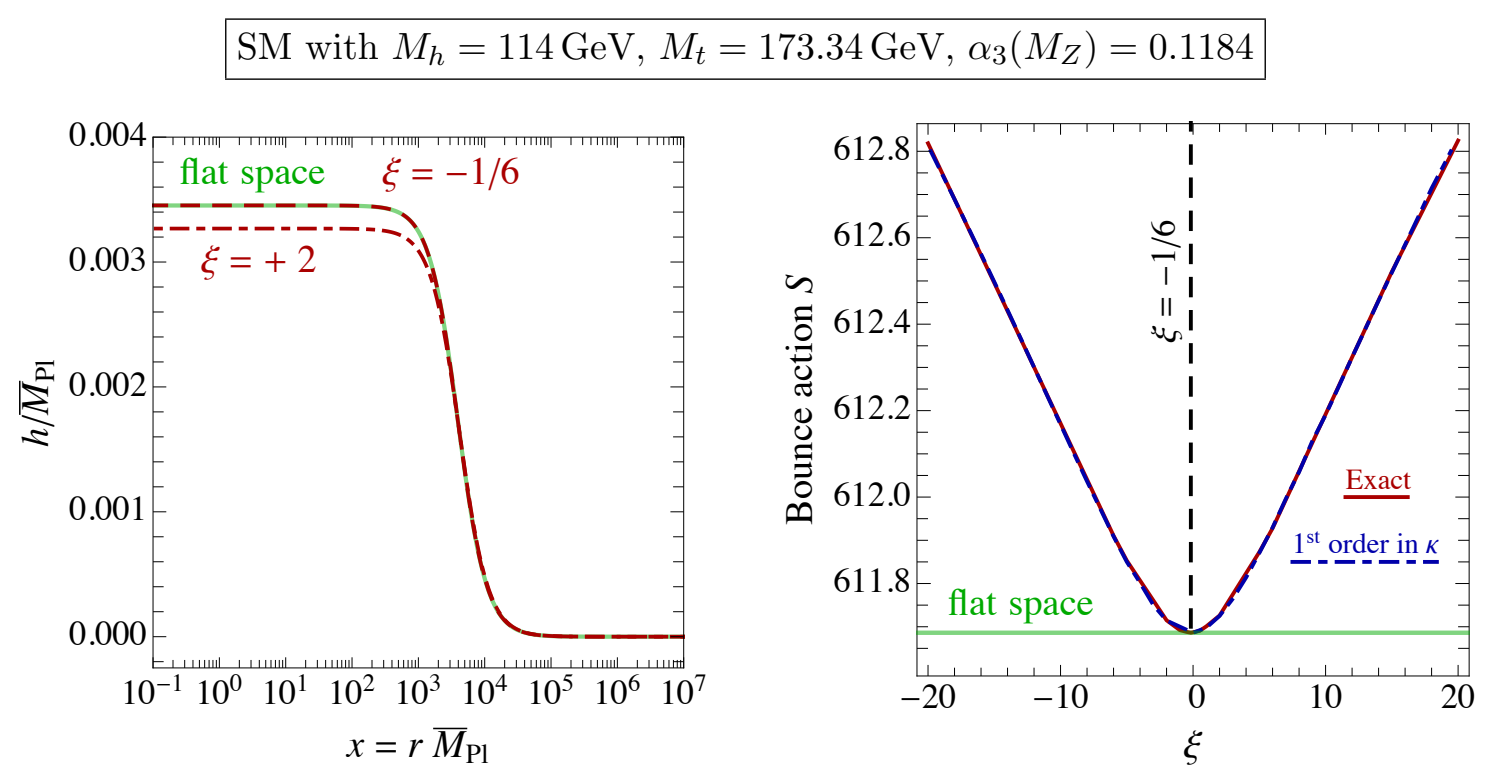

Figure 2. SM bounce solutions for different values of $\xi$ (left panel), and their action (right panel). We consider $M_{h}=114 \mathrm{GeV}$, which is the value that saturates the meta-stability bound for the central value of the top mass. The bounce is sub-Planckian, so that gravitational corrections can be computed perturbatively.

For the following we concentrate on a non-minimal coupling of the form $f(h)=\xi h^{2}$. For a scale-invariant potential $V(h)=\lambda h^{4} / 4$ with $\lambda<0$, and neglecting gravity, the bounce $h_{0}(r)$ can be computed analytically. It depends on an arbitrary scale $R$ :

$$
h_{0}(r)=\sqrt{\frac{2}{|\lambda|}} \frac{2 R}{r^{2}+R^{2}} .
$$

Quantum and gravitational corrections can be computed perturbatively by expanding around the solution of eq. (2.12). Eq. (2.10) becomes

$$
\rho_{1}^{\prime}=\frac{8 r^{2} R^{2}}{3|\lambda|\left(r^{2}+R^{2}\right)^{3}}(1+6 \xi)
$$

Making use of eq. (2.9) we obtain the final result:

$$
S=\min _{R}\left(\frac{8 \pi^{2}}{3|\lambda(\bar{\mu})|}+\Delta S_{\text {quantum }}+\Delta S_{\text {gravity }}\right), \quad \Delta S_{\text {gravity }}=\frac{32 \pi^{2}(1+6 \xi)^{2}}{45\left(R \bar{M}_{\mathrm{Pl}} \lambda\right)^{2}} .
$$

The gauge-invariant quantum correction $\Delta S_{\text {quantum }}$ has been computed in [32] at one loop in the $\overline{\mathrm{MS}}$ scheme. It compensates for the RGE-scale dependence of $\lambda$, such that one can conveniently choose the RGE scale $\bar{\mu}=1 / R^{3}$ The gravitational correction at leading

\footnotetext{
${ }^{3}$ The one-loop calculation of the decay rate basically amounts to substituting the tree-level action with the one-loop action. The path-integral over all fluctuations has been computed in [32] up to the last $\int d \ln R e^{-S(R)}$ integral over dilatations, which is a higher-order effect because the SM tree-level action is scale-invariant. The SM running of $\lambda$ fixes the intermediate value of $R$ that dominates the integral. We here adopt the simple Gaussian approximation such that $\int d \ln R e^{-S(R)}$ becomes $\min _{R} e^{-S(R)}$, namely the least action principle.
} 
SM with $M_{h}=125.09 \mathrm{GeV}, M_{t}=173.34 \mathrm{GeV}, \alpha_{3}\left(M_{Z}\right)=0.1184$
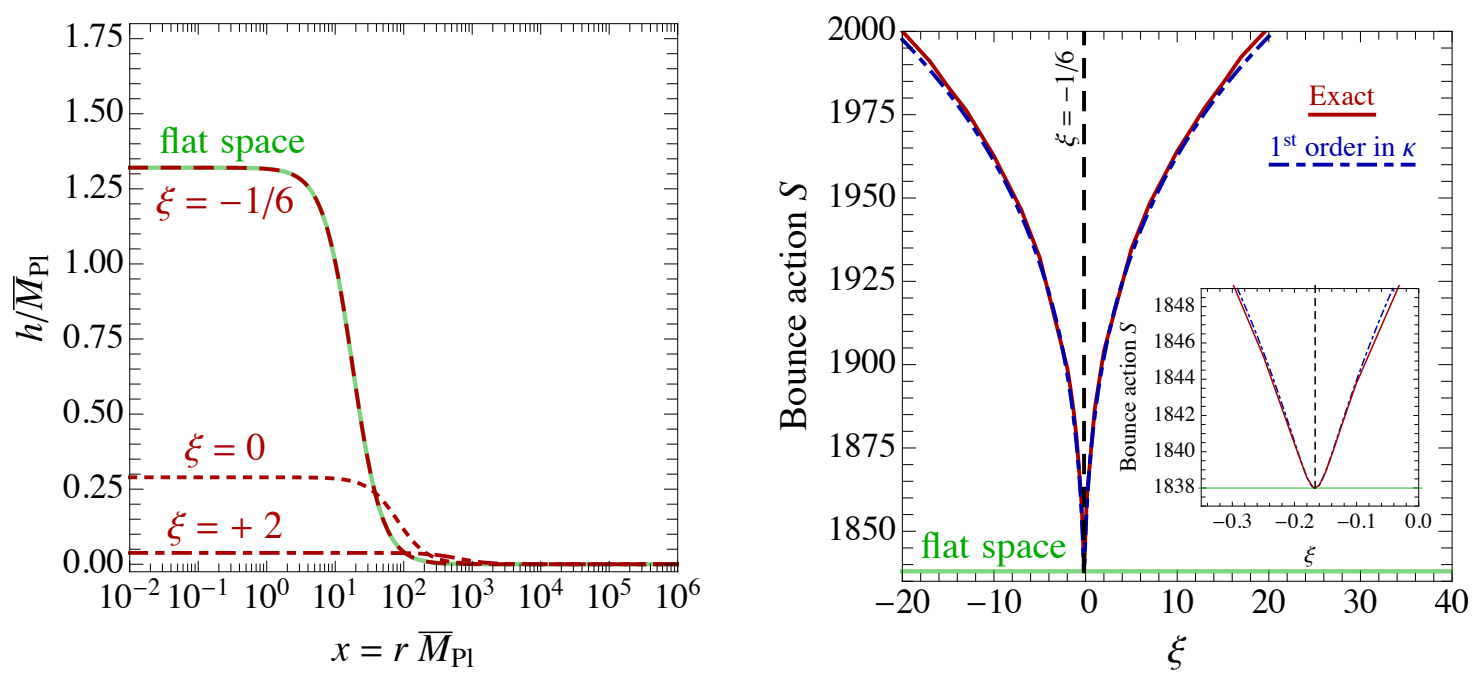

Figure 3. SM bounce solutions for different values of $\xi$ (left panel), and their action (right panel). We consider here the best fit Higgs mass $M_{h}=125.09 \mathrm{GeV}$, for which the vacuum decay rate is negligibly small. For ease of visualisation we do not consider uncertainties due to higher order corrections.

order in $1 / M_{\mathrm{Pl}}, \Delta S_{\text {gravity }}$, agrees with [4]. We included here the full quadratic dependence of $\Delta S_{\text {gravity }}$ on $\xi$, going beyond the linear order in $\xi$ computed in [4]. Furthermore, $S$ indirectly acquires a different dependence on $\xi$ in view of the minimisation over $R$ dictated by eq. (2.14).

Figure 2 demonstrates that the full numerical result agrees with the approximate expression. We considered $M_{h}=114 \mathrm{GeV}$, which is the value that saturates the meta-stability bound for the central value of the top quark mass. The bounce is sub-Planckian, such that gravitational corrections are small and can be reliably computed. ${ }^{4}$ Keeping instead $M_{h}$ at its experimental value and raising $M_{t}$ up to its meta-stability boundary $M_{t} \lesssim 178 \mathrm{GeV}$ again leads to a sub-Planckian bounce, with $h(0) \sim 0.1 \bar{M}_{\mathrm{Pl}}$.

\footnotetext{
${ }^{4}$ The authors of [10] justify their criticism by claiming that no first-order correction $h_{1}(r)$ to the bounce with the correct boundary conditions $h_{1}^{\prime}(0)=0$ and $h_{1}(\infty)=0$ exists. While their calculation is technically correct, they miss the crucial physical point. Indeed, they expand around the solution $h_{0}(r)$ of eq. (2.12), which corresponds to the tree-level SM action that is scale-invariant and thereby does not determine the scale $R$ of the bounce. Adding only the effect of either gravity (operators with negative mass dimension) or a Higgs mass term (operators with positive mass dimension) results in either $R \rightarrow \infty$ or $R \rightarrow 0$ : namely the bounce no longer exists. The problematic $h_{1}$ is another manifestation of this issue. In the real physical problem the bounce exists because quantum corrections break scale invariance selecting an intermediate finite value for the bounce scale $R$, roughly given by the inverse scale that minimizes the running $\lambda$. Therefore, the correct physical procedure is the one followed in [4], and summarised here in eq. (2.14): compute the quantum corrections to the action as a function of $R$, and use them to determine $R$. The gravitational corrections can then be computed perturbatively. The solution for $h_{1}(r)$ is not needed in this calculation, but can be computed from the quantum-corrected potential - or any potential that fixes a scale for $h_{0}(r)$. The equation for $h_{1}(r)$ then has a solution that satisfies the correct boundary conditions, thus resolving the issue raised in [10].
} 

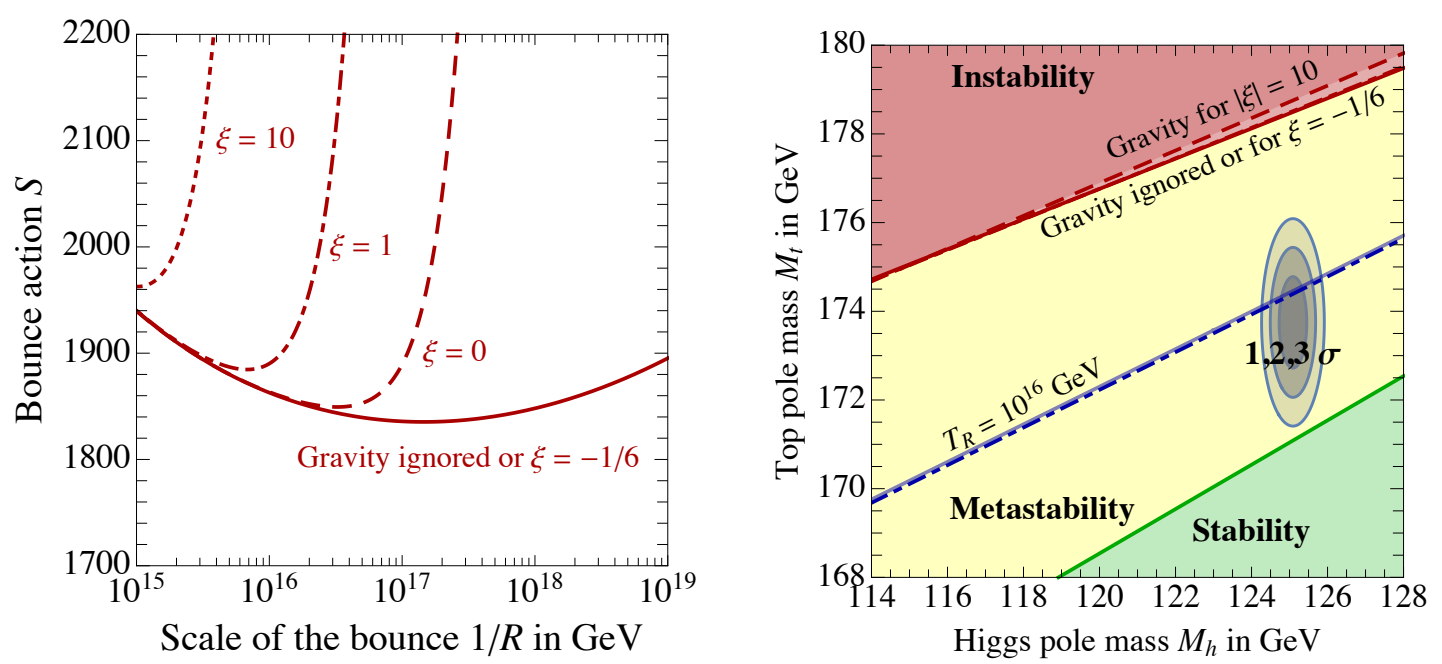

Figure 4. Left: bounce action as function of $R$ for $M_{h}=125.09 \mathrm{GeV}$. Right: SM phase diagram for $\alpha_{3}\left(M_{Z}\right)=0.1184$. The continuous red line is obtained ignoring gravitational corrections or including them assuming the conformal value $\xi=-1 / 6$ of the Higgs coupling to gravity; the almost coincident dot-dashed line assumes $\xi=0$; the dashed line assumes $|\xi| \sim 10$. The ellipses show the

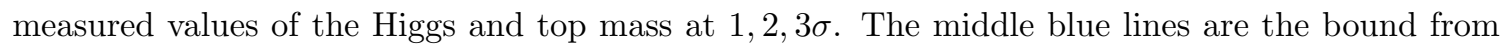
thermal tunneling, assuming a reheating temperature of $10^{16} \mathrm{GeV}$.

In figure 3 we consider the central value $M_{h} \approx 125.09 \mathrm{GeV}$, which leads to a negligibly small vacuum decay rate dominated by a bounce with Planck-scale size, $h(0) \sim M_{\mathrm{Pl}}{ }^{5}$ Naively, this is beyond the applicability domain of the low-energy expansion of [4]. Nevertheless, the analytic approximation agrees well with the full numerical result because approximated scale invariance combined with the positivity of $\Delta S_{\text {gravity }}$ implies that vacuum decay is dominated by bounces with $h(0) \sim 1 / R$ small enough not to be suppressed by gravity, as illustrated in the left panel of figure 4 .

The right panel of figure 4 shows the SM phase diagram in the $\left(M_{h}, M_{t}\right)$ plane for $\alpha_{3}\left(M_{Z}\right)=0.118$. We used our numerical code; the difference with respect to the analogous plot obtained from the analytic expression is as small as unknown quantum-gravity effects. We see that gravitational corrections have a minor effect: the upper dashed line is obtained for $|\xi|=10$, and it differs by $\approx 0.5 \mathrm{GeV}$ in $M_{t}$ from the dot-dashed line, obtained for $\xi=0$. In turn, it is almost coincident with the continuous line, obtained either setting $\xi=-1 / 6$ or ignoring gravity.

This last feature is understood noticing that $\Delta S_{\text {gravity }}$ vanishes for the conformal value $\xi=-1 / 6$. This equality is not limited to the leading order in $1 / M_{\mathrm{Pl}}$ : the Fubini bounce of eq. (2.12), together with the flat metric $\rho(r)=r$, is an exact bounce solution of the full gravitational problem for $\xi=-1 / 6$ and constant negative $\lambda$, such that the bounce action is the same as in the non-gravitational case. In particular, the last term in eq. (2.4) identically vanishes. Indeed, for $\xi=-1 / 6$, the Ricci scalar reduces to the simple form

\footnotetext{
${ }^{5}$ For the sake of comparison, we explain the discrepancy between our figure 3 and the analogue plot in [10]: they use the tree-level quartic potential $\frac{1}{4} \lambda h^{4}$, while we use the 2-loop SM effective potential [2]. Both computations use 3-loop RGE running in the SM.
} 
$\mathcal{R}=\kappa[4 V-h d V / d h]$, which vanishes for a scale-invariant potential $V=\lambda h^{4} / 4$. These properties can be also derived without any explicit computation from symmetry arguments: for $\xi=-1 / 6$ the Higgs Lagrangian is conformally invariant; one can rescale the metric so that any conformally flat metric, such as the one we consider in eq. (2.2), is equivalent to the flat metric. Thus, any solution of the $\lambda h^{4}$-theory on flat space-time is also a solution when gravitational effects are included. ${ }^{6}$

\subsection{Effects of new Planckian physics}

Even if $h_{0}(0) \ll M_{\mathrm{Pl}}$, Planck suppressed operators such as $|H|^{6} / M_{\mathrm{Pl}}^{2}$ and $|H|^{2}\left|D_{\mu} H\right|^{2} / M_{\mathrm{Pl}}^{2}$ give extra corrections to the bounce action of the same order as gravitational corrections: at leading order in $1 / M_{\mathrm{Pl}}$ they can be incorporated in $\xi$ through field redefinitions of the Higgs and of the graviton [4]. Both $\xi$, as well as such effective operators, are unavoidably generated when quantum corrections are added to the Einstein-Hilbert-Higgs action. However, at higher orders an increasingly larger number of effective operators enters the game, and the effective-theory expansion breaks down.

In order to compute if gravity suppresses or enhances vacuum decay, one needs the theory of gravity, which is unknown. Assuming relativistic invariance, general arguments tell that such unknown theory must either contain an infinite number of positive-norm fields (possibly resulting from some string theory) or a four-derivative graviton which includes one negative norm component (see [33-35] for attempts to find a sensible quantum interpretation).

The string solution suggests a complicated unknown landscape of extra negative-energy AdS minima, and thereby new contributions to vacuum decay. As far as vacuum decay is concerned, the main implication of such landscape are captured by adding one new scalar $s$, possibly with Planckian mass and decoupled from the Higgs. Tunnelling along the $s$ direction opens a new channel for vacuum decay. Its rate can be arbitrarily fast, independently of the mass of $s$. This issue is orthogonal to SM vacuum decay: Planckscale physics cannot suppress sub-Planckian contributions to SM vacuum decay, which can only be affected by new physics at lower energies. In summary, calculations of the SM vacuum decay rate hold up to the caveat 'unless extra Planck-scale vacuum decay destroys the universe earlier', analogously to how computations of the life-time of SM particles hold up to the same obvious caveat, which is conveniently left implicit. ${ }^{7}$

The second solution, which we refer to as "agravity", gives more precise conclusions. The Euclidean Einstein-Hilbert-Higgs action is replaced by

$$
S=\int d^{4} x \sqrt{g}\left[\frac{\left(\partial_{\mu} h\right)\left(\partial^{\mu} h\right)}{2}+V(h)-\frac{\mathcal{R}}{2 \kappa}-\frac{\mathcal{R}}{2} \xi h^{2}-\frac{\mathcal{R}^{2}}{6 f_{0}^{2}}+\frac{\mathcal{R}_{\mu \nu}^{2}-\mathcal{R}^{2} / 3}{f_{2}^{2}}\right],
$$

\footnotetext{
${ }^{6}$ The fact that the Einstein-Hilbert term breaks conformal invariance does not invalidate this conclusion. To show this consider conformal gravity (i.e. replace the Einstein-Hilbert term by the square of the Weyl tensor). The full theory is now conformally invariant. Any solution of the $\lambda h^{4}$-theory on flat space-time is also a solution when gravitational effects are included. This implies in particular that the energy momentum tensor is zero for such a solution (recall that the Weyl tensor vanishes on flat space-time) and $\xi=-1 / 6$. So this configuration is also a solution of the Einstein-Hilbert-Higgs field equations.

${ }^{7}$ The authors of [36] emphasize that Planck-scale physics can give extra contributions to vacuum decay, but proposing a specific example which relies on an uncontrolled expansion in $1 / M_{\mathrm{Pl}}$ : an extra Planck-scale minimum in the Higgs potential obtained by adding terms $-h^{6} / M_{\mathrm{Pl}}^{2}$ and $+h^{8} / M_{\mathrm{Pl}}^{4}$.
} 
where $f_{0}, f_{2}, \xi$ are dimensionless gravitational couplings, such that theory is renormalizable. The term suppressed by $f_{2}$ gives rise to a ghost state which might admit a sensible physical interpretation [33-35, 37]. In any case, this term does not contribute to the bounce action, because it is the square of the conformally-invariant Weyl tensor, up to total derivatives, and our background is conformally flat. The equation of motion for $h$ and the expression for $\mathcal{R}=-6\left(\rho^{2} \rho^{\prime \prime}+\rho \rho^{2}-\rho\right) / \rho^{3}$ remain unchanged while the equation for $\rho(r)$ becomes

$$
\rho^{\prime 2}=1+\frac{\kappa \rho^{2}}{3\left[1+\kappa\left(\xi h^{2}+2 \mathcal{R} / 3 f_{0}^{2}\right)\right]}\left[\frac{h^{\prime 2}}{2}-V-\frac{\mathcal{R}^{2}}{6 f_{0}^{2}}-3 \frac{\rho^{\prime}}{\rho}\left(2 \xi h h^{\prime}+\frac{2 \mathcal{R}^{\prime}}{3 f_{0}^{2}}\right)\right] .
$$

This equation can be obtained from the $r r$ component of the Einstein equations. For our present purposes, it is convenient to ignore it, and rather close the system by adding the trace of the Einstein equations:

$$
\left(\frac{\bar{M}_{\mathrm{Pl}}^{2}+\xi h^{2}}{2}-\frac{\square}{f_{0}^{2}}\right) \mathcal{R}=\frac{h^{2}}{2}+2 V+\frac{3 \xi}{2} \square h^{2},
$$

where $\square$ is the covariant d'Alambertian. This allows to read the main qualitative difference between agravity and Einstein gravity. At energies much smaller than $f_{0} \bar{M}_{\mathrm{Pl}}$ the new $\square$ term is irrelevant and one recovers the Einstein limit. At larger energies, the $\square$ term suppresses $\mathcal{R}$ with respect to the Einstein limit, so that the gravitational correction to the bounce action saturates at $\left|\Delta S_{\text {gravity }}\right| \lesssim \pi^{2} f_{0}^{2} / \lambda^{2}$. This means that gravitational corrections to $\mathrm{SM}$ vacuum decay can be ignored if $f_{0}$ is numerically small as in [34]. A negative value of $\xi$ (such that the Einstein term vanishes for $h=\bar{M}_{\mathrm{Pl}} / \sqrt{-\xi}$ ) opens a new vacuum instability.

The only solid conclusion that one can draw from the above considerations is that new Planck-scale physics cannot cure the SM Higgs vacuum instability, if such instability appears much below $\bar{M}_{\mathrm{Pl}}$.

\section{SM vacuum decay at finite temperature}

The instability of the SM potential can also give rise to thermal tunneling in the early universe, if it went through a hot enough phase (cosmological data only imply that the universe has been hotter than a few $\mathrm{MeV}$ ). The space-time probability density of thermal tunneling at temperature $T$ is given by

$$
\gamma=\frac{d \wp}{d^{4} x} \approx T^{4}\left(\frac{S}{2 \pi}\right)^{3 / 2} e^{-S}
$$

where $S(T)=\int_{0}^{1 / T} d t_{E} \int d^{3} x \mathscr{L}$ is the action of the thermal bounce at temperature $T$, which is a solution to the classical equations of motion with periodicity $1 / T$ in Euclidean time $t_{E}$. The total cosmological probability of thermal tunneling up to today is obtained by integrating over the past light-cone

$$
\wp=\int d t d V \gamma=V_{0} \int d t a^{3} \gamma
$$

where $V_{0}=4 \pi\left(3.4 / H_{0}\right)^{3} / 3$ is the volume within the present horizon and $a$ is the Universe scale factor, equal to one today at $t=t_{0}$. Using conservation of entropy to relate $a$ to $T$ 

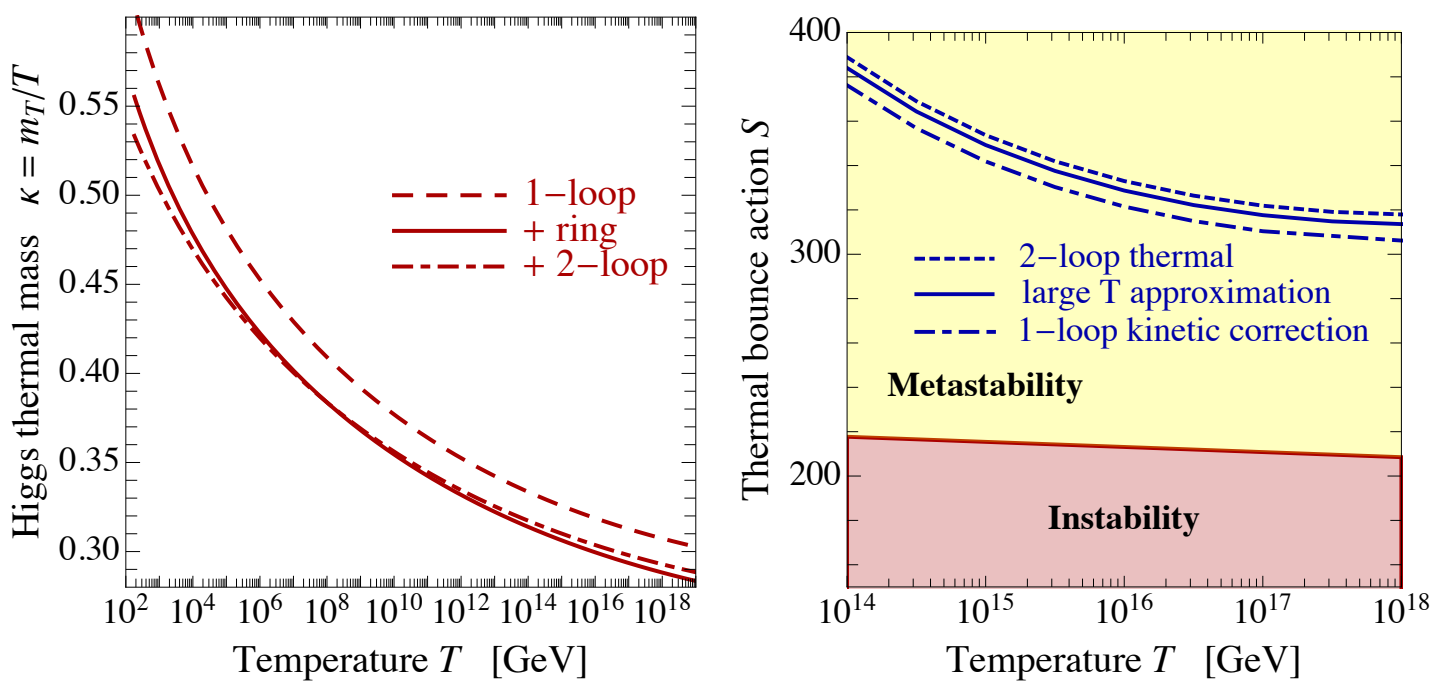

Figure 5. We consider the SM for $M_{h}=125.09 \mathrm{GeV}, M_{t}=173.34 \mathrm{GeV}, \alpha_{3}\left(M_{Z}\right)=0.1184$. Left: Higgs thermal mass $m_{T} / T$ as function of the temperature, as precisely defined in eq. (3.5), computed adding higher order corrections in the thermal loops. Right: action of the thermal bounce $S(T)$ computed with the usual large-temperature approximation (solid curve), adding 2-loop thermal masses (dotted), 1-loop kinetic corrections (dot-dashed). We also show the boundary between stability and meta-stability.

we get

$$
\wp \approx \frac{\sqrt{2} V_{0}}{\sqrt{\Omega_{\gamma}} H_{0}} \frac{g_{* S 0}}{g_{*}^{3 / 2}} \int \frac{d T}{T}\left(\frac{T_{0}}{T}\right)^{5} \gamma \approx 117 \int \frac{d T}{T}\left(\frac{T_{0}}{T}\right)^{5} \frac{\gamma}{H_{0}^{4}}
$$

where $T_{0}=2.7 \mathrm{~K}$ is the present temperature, $H_{0} \approx 67.4 \mathrm{~km} / \mathrm{sec} \mathrm{Mpc}$ is the present Hubble rate, $g_{* S 0}=3.94$ the total number of effective degrees of freedom contributing to the entropy after $e^{+} e^{-}$annihilation, and $g_{*}=106.75$ the number of SM degrees of freedom at $T$ much larger than the electro-weak scale, when the thermal probability receives the dominant contribution. A small probability of thermal tunnelling $\wp \ll 1$ is roughly obtained if $S(T) \gtrsim 206+\ln \left(M_{\mathrm{Pl}} / T\right)$ at any $T$ below the reheating temperature.

In the following we revisit computations of the thermal tunnelling rate adding three new effects to previous computations. In section 3.1 we include two-loop corrections to the thermal Higgs potential. In section 3.2 we include one-loop derivative corrections to the thermal Higgs action. In section 3.3 we explore time-dependent bounces.

\subsection{Two-loop Higgs thermal mass}

The temperature-dependent effective potential can be expanded as

$$
\begin{aligned}
V_{\text {eff }}(h, T)= & V_{0}(h)+V_{1-\text { loop }}(h)+V_{2-\text { loop }}(h) \\
& +V_{1-\text { loop }}(h, T)+V_{\text {ring }}(h, T)+V_{2-\text { loop }}(h, T) \cdots,
\end{aligned}
$$

where the first three terms refer to $T=0$. To make the structure of the effective potential more transparent, a reasonable approximation is

$$
V_{\mathrm{eff}}(h, T) \approx m_{T}^{2}(h) \frac{h^{2}}{2}+\frac{\lambda_{\mathrm{eff}}(h)}{4} h^{4} .
$$


The effective quartic coupling $\lambda_{\text {eff }}$ is extracted from the RG-improved effective potential at two-loop order and zero temperature. The two-derivative Higgs kinetic term is canonically normalized, up to corrections not enhanced by large logarithms. We write the Higgs thermal mass as $m_{T}^{2} \equiv \kappa^{2} T^{2}$ with $\kappa^{2}=\kappa_{1-\text { loop }}^{2}+\kappa_{\text {ring }}^{2}+\kappa_{2-\text { loop }}^{2}$ and

$$
\begin{aligned}
\kappa_{1-\text { loop }}^{2} & =\frac{1}{16} g^{\prime 2}+\frac{3}{16} g^{2}+\frac{1}{4} y_{t}^{2}+\frac{1}{2} \lambda \\
\kappa_{\text {ring }}^{2} & =-\frac{1}{16 \pi} \sqrt{\frac{11}{6}}\left(g^{\prime 3}+3 g^{3}\right)-\frac{3 \lambda}{8 \pi}\left(g^{\prime 2}+3 g^{2}+8 \lambda+4 y_{t}^{2}\right)^{1 / 2} .
\end{aligned}
$$

Higher-order corrections to $\kappa$ are given in [38, 39], and contain logarithmic factors that cancel the dependence on the RG-scale $\bar{\mu}$ of the lower-order terms, roughly dictating that the running couplings in eq. (3.6) are renormalised at $\bar{\mu} \sim T$. We fix the residual RG-scale dependence setting $\bar{\mu}=T ; m_{T}^{2}$ acquires a logarithmic dependence on $h$, and in the left panel of figure 5 we plot its value at the relevant scale $h=T$. We see that the 2-loop contribution is small.

In the right panel of figure 5 we show that including the 2-loop thermal mass gives a small correction to the bounce action, at the few $\%$ level. This is consistent with the fact that the 2-loop correction to $\kappa$ is small and that the bounce action is roughly proportional to $\kappa$ (if the full thermal potential is approximated as a constant $m_{T}$ and a constant $\lambda_{\text {eff }}$ the bounce action is $\left.S \approx 6.015 \pi \kappa / \lambda_{\text {eff }}[27]\right)$.

\subsection{One-loop thermal correction to the Higgs kinetic energy}

Various authors computed the one-loop thermal potential. However, the bounce action receives comparable contributions from the kinetic part of the Lagrangian. The computation of vacuum decay at $T=0$ has been performed including the full one-loop effective action [32], which includes an infinite number of derivatives. Performing similar computations at finite $T$ is more difficult: we study here the impact of thermal corrections to the two-derivative Higgs kinetic term.

One-loop thermal corrections to derivative terms in the effective action at finite temperature were presented in $[40,41]$ and are of relative order $g^{2} / 4 \pi$. We can focus on spatial derivatives, because they receive the main correction in the large $T$ limit and because the thermal bounce is time-independent (see section 3.3). Such corrections can be written as

$$
\Delta S=\frac{1}{2} \int_{0}^{1 / T} d t_{E} \int d^{3} x Z_{2}(h, T)\left(\partial_{i} h\right)^{2}
$$

where $i$ runs over spatial coordinates. In the SM at large temperature $Z_{2}$ is given by

$$
\begin{aligned}
Z_{2}(h, T) \approx & \frac{T}{4 \pi}\left\{\frac{\lambda^{2} h^{2}}{4}\left[\frac{3}{m_{h}^{3}(T)}+\frac{1}{m_{\chi}^{3}(T)}\right]-\frac{4 g^{2}}{3}\left[\frac{1}{m_{\chi}(T)+m_{W}}\right]\right. \\
& -\frac{2 g^{2}}{3 c_{\mathrm{W}}^{2}}\left[\frac{1}{m_{\chi}(T)+m_{Z}}\right]+\frac{g^{2} m_{W}^{2}}{12}\left[\frac{1}{2 m_{W_{L}}^{3}(T)}+\frac{5}{m_{W}^{3}}\right] \\
& \left.+\frac{g^{2} m_{Z}^{2}}{24}\left[\frac{c_{\theta}^{2}}{2 m_{Z_{L}}^{3}(T)}+\frac{5}{m_{Z}^{3}}\right]+\frac{g^{2} m_{Z}^{2}}{24}\left[\frac{s_{\theta}^{2}}{2 m_{\gamma_{L}}^{3}(T)}+\frac{8 s_{\theta} c_{\theta}}{\left(m_{Z_{L}}(T)+m_{\gamma_{L}}(T)\right)^{3}}\right]\right\} .
\end{aligned}
$$


Thermal masses $m_{i}^{2}(T)=m_{i}^{2}+\kappa_{i}^{2} T^{2}$ for $i=h, \chi, W_{L}, W_{T}, Z_{T}, \gamma_{T}$ can be computed in terms of the usual field-dependent zero-temperature mass $m_{i}$, and of [42]

$$
\kappa_{h}=\kappa_{\chi}=\frac{3 g^{2}+g^{\prime 2}}{16}+\frac{\lambda}{2}+\frac{y_{t}^{2}}{4}, \quad \kappa_{W_{L}}=\frac{11}{6} g^{2}, \quad \kappa_{W_{T}}=\kappa_{Z_{T}}=\kappa_{\gamma_{T}}=0 .
$$

The masses $m_{Z_{L}}$ and $m_{\gamma_{L}}$ are the eigenvalues of the thermal mass matrix [42]

$$
\left(\begin{array}{cc}
m_{Z_{L}}^{2}(T) & 0 \\
0 & m_{\gamma_{L}}^{2}(T)
\end{array}\right)=R\left(\begin{array}{cc}
m_{Z}^{2}+\Pi_{Z_{L} Z_{L}}(T) & \Pi_{Z \gamma_{L}}(T) \\
\Pi_{Z \gamma_{L}}(T) & \Pi_{\gamma_{L} \gamma_{L}}(T)
\end{array}\right) R^{T}, \quad R=\left(\begin{array}{cc}
c_{\theta} & -s_{\theta} \\
s_{\theta} & c_{\theta}
\end{array}\right),
$$

where $R$ is the matrix that rotates the mass eigenstates at $T=0$ into those at $T \neq 0$, which is defined in terms of a mixing angle $\theta\left(c_{\theta} \equiv \cos \theta, s_{\theta} \equiv \sin \theta\right)$, and

$$
\begin{aligned}
\Pi_{Z_{L} Z_{L}}(T) & =\left[\frac{2}{3} g^{2} c_{\mathrm{W}}^{2}+\frac{g^{2}}{6 c_{\mathrm{W}}^{2}}\left(1-2 s_{\mathrm{W}}^{2} c_{\mathrm{W}}^{2}\right)+\frac{g^{2}}{c_{\mathrm{W}}^{2}}\left(1-2 s_{\mathrm{W}}^{2}+\frac{8}{3} s_{\mathrm{W}}^{4}\right)\right] T^{2}, \\
\Pi_{\gamma_{L} \gamma_{L}}(T) & =\frac{11}{3} e^{2} T^{2}, \\
\Pi_{Z \gamma_{L}}(T) & =\frac{11}{6} e g \frac{c_{\mathrm{W}}^{2}-s_{\mathrm{W}}^{2}}{c_{\mathrm{W}}} T^{2}
\end{aligned}
$$

where $c_{\mathrm{W}} \equiv \cos \theta_{W}$ and $s_{\mathrm{W}} \equiv \sin \theta_{W} . Z_{2}(h, T)$ was presented previously in the $g_{Y}=0$ limit in [41]. Here we also included the effect of $g_{Y}$. In this formula we only included the dominant contribution of the zero Matsubara modes of bosons: in this approximation there are no corrections induced by the top-quark Yukawa coupling.

Up to higher orders term, the correction to the bounce action is given by the new term, eq. (3.8), evaluated along the bounce computed ignoring it. We find that the bounce action changes at the few $\%$ level, see figure 5 .

We do not compute the effect of terms with more than 2 derivatives, but we estimate that they can give effects comparable to the effect of corrections to the 2 derivative term. Indeed, loop corrections give higher-order Higgs derivative terms, which can be large when the Higgs has a sizeable coupling to some other particle not much heavier than the Higgs itself. At zero temperature, all masses come from the Higgs vev: in the limit of a large vev the Higgs is relatively lighter than $t, W, Z$, because its mass is controlled by the Higgs self-coupling $\lambda$, which runs to relatively small values at large energy. As a consequence, at $T=0$ and large vev one has $m_{h} \ll m_{t, W, Z}$, so that higher-order derivative terms are suppressed. At finite temperature the Higgs receives an extra thermal mass given by the larger $y_{t}, g_{1}, g_{2}$ couplings: as a consequence all thermal masses are comparable, and higher-order derivative terms could be significant.

\subsection{Is the thermal bounce time-independent?}

The thermal tunneling rate at temperature $T$ is computed from the action

$$
S=4 \pi \int_{0}^{\beta} d t_{E} \int_{0}^{\infty} d r r^{2}\left[\frac{1}{2}\left(\frac{\partial h}{\partial t_{E}}\right)^{2}+\frac{1}{2}\left(\frac{\partial h}{\partial r}\right)^{2}+V_{\text {eff }}(h)\right]
$$



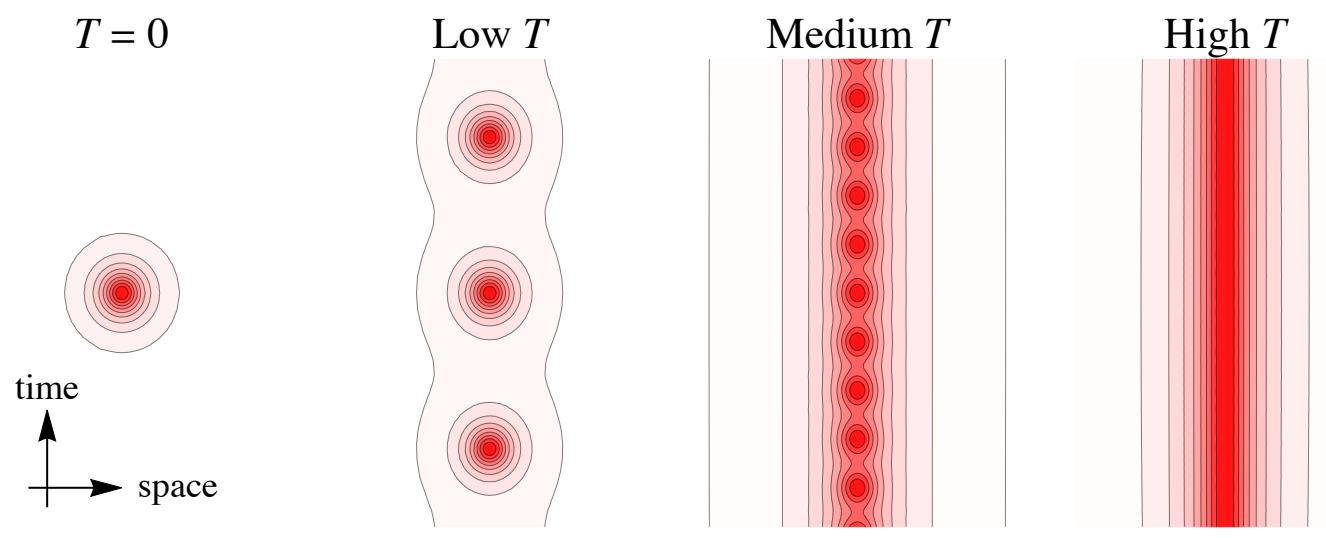

Figure 6. Bounces at different temperatures. The vertical axis represents the Euclidean time direction, and the horizonal axis represents the spatial radius. At $T=0$ (left-most panel) the bounce solution enjoys an $\mathrm{O}(4)$ symmetry. At finite temperature, the bounce solution becomes a series of bubbles placed at distance $1 / T$ in the time direction. At large temperature (right-most panel) the bounce no longer depends in time.

of a bounce $h\left(r, t_{E}\right)$ where $r \equiv \sqrt{|\vec{x}|^{2}}$ is the spatial radius and $t_{E}=-i t$ is Euclidean time. The bounce solves the classical equation

$$
\frac{\partial^{2} h}{\partial t_{E}^{2}}+\frac{\partial^{2} h}{\partial r^{2}}+\frac{2}{r} \frac{\partial h}{\partial r}=\frac{d V_{\text {eff }}}{d h}
$$

with modified boundary conditions

$$
\left.\frac{\partial h}{\partial t_{E}}\right|_{t_{E}=0, \pm 1 / 2 T}=0,\left.\quad \frac{\partial h}{\partial r}\right|_{r=0}=0, \quad \lim _{r \rightarrow \infty} h\left(r, t_{E}\right)=0
$$

that impose periodicity in Euclidean time, $h\left(r, t_{E}+\beta\right)=h\left(r, t_{E}\right)$.

One trivial solution is a bounce constant in time, and normally this is the lowestaction solution at large enough temperature as illustrated in the right panel of figure 6 (see also [43-45]). Indeed, when a theory has a characteristic energy scale $m$, it sets the scale of the $\mathrm{O}(4)$-symmetric bounce valid at $T=0$. At low $T$ periodicity is irrelevant, because the time period is much slower than the scale of the $T=0$ bounce, as illustrated in the left panel of figure 6 . For $T$ much larger than the scale of the $T=0$ bounce, the short time periodicity implies that (if the vacuum instability still exits) the bounce becomes constant in time. Thereby the action of the time-independent bounce scales as $S \propto 1 / T$ and is given by $S \sim m / T$ such that it dominates tunnelling above some critical temperature of order $m$.

Previous computations of thermal decay in the SM at $T \gg M_{h}$ assumed a timeindependent thermal bounce. However, the physical Higgs mass $M_{h}$ is not the relevant energy scale for the instability of the SM Higgs potential. Rather, $M_{h}$ can be neglected, obtaining a quasi-scale invariant action for the Higgs. The assumption that $T$ is much larger than the energy scale of the problem must be reconsidered, in view of the fact that the problem does not have a characteristic energy scale.

In the thermal bath, $h$ acquires a thermal mass $m_{T}=\kappa T$. Therefore, the large temperature limit $T \gg m_{T}$ would correspond to $\kappa \ll 1$ and would give a constant $S \sim$ 


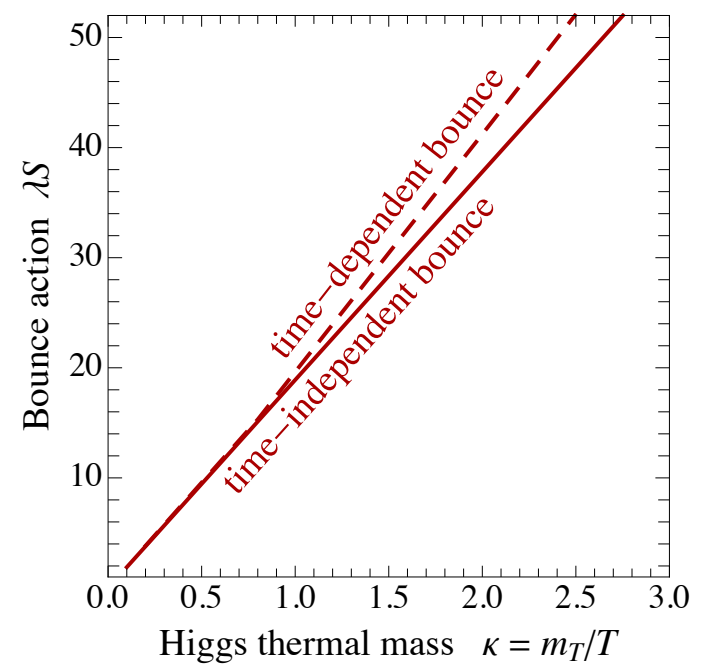

Figure 7. Rescaled thermal bounce action $\lambda S$ as a function of the Higgs thermal mass $\kappa=m_{T} / T$. The solid (dashed) line corresponds to the time-independent (time-dependent) bounce.

$m_{T} / T=\kappa$. The SM predicts $\kappa \sim g \sim 0.4$, see eq. (3.6) and figure 5 : it is not much smaller than unity, potentially threatening the validity of usual computations that assume a time-independent thermal bounce. In order to settle the issue, we investigate whether time-dependent bounces have lower action.

To start we consider a simplified SM-like potential $V_{\text {eff }}(h)=\frac{1}{2} \kappa^{2} T^{2} h^{2}-\frac{1}{4} \lambda h^{4}$ with constant $\kappa$ and $\lambda$. By rescaling $h(x)$ to a dimensionless $\eta(\xi)$ defined by $h(x)=\eta(\xi) \kappa T / \sqrt{\lambda}$ and $x_{\mu}=\xi_{\mu} / \kappa T$ (we denote as $\tau$ and $\rho$ the dimensionless time and radius) the action becomes

$$
S=\frac{4 \pi}{\lambda} \int_{0}^{\kappa} d \tau \int_{0}^{\infty} d \rho \rho^{2}\left[\frac{1}{2}\left(\frac{\partial \eta}{\partial \tau}\right)^{2}+\frac{1}{2}\left(\frac{\partial \eta}{\partial \rho}\right)^{2}+\frac{1}{2} \eta^{2}-\frac{1}{4} \eta^{4}\right]
$$

This shows that $\lambda S$ does not depend on $\lambda$ and that for the time-independent bounce, $\lambda S$ is proportional to $\kappa$. The precise result is $\lambda S=6.015 \pi \kappa$ [27]. The rescaled action $\lambda S$ of a time-dependent bounce can be a more generic function of $\kappa$. Figure 7 shows our numerical result for $\lambda S$, finding that the time-dependent bounce always has a higher action and is thereby subdominant. ${ }^{8}$

We next consider the full SM thermal potential: the bounce action can significantly deviate from the above approximation, but again the time-independent bounce dominates.

\footnotetext{
${ }^{8}$ Solving numerically the differential equation eq. (3.15) is not an easy task, since it is a non-linear equation with non-trivial boundary conditions in space and time. We discretise it on a space-time lattice, obtaining an ordinary non-linear equation $E_{i}=0$ at each point $i$. Next, we numerically minimise $\sum E_{i}^{2}$ applying the usual Newton-like methods. These need a starting ansatz, and convergence is obtained provided that the starting point is good enough. Appropriate choices are the $\mathrm{O}(4)$-symmetric bounce, or even the $T=0$ bounce of eq. (2.12), provided that $h(0,0)$ is left as a free parameter. Linear equations, such as boundary conditions, can be first imposed exactly, improving the procedure.
} 


\section{Conclusions}

We reconsidered quantum and thermal vacuum decay in the SM.

Concerning vacuum decay, we validated the semi-analytic low-energy approximation for gravitational corrections at leading-order in 1/ $M_{\mathrm{Pl}}$ proposed in [4] (and wrongly criticized in $[7,10])$ through numerical computations in a toy model (section 2.2) and in the SM (section 2.3). We generalised [4] allowing for a non-minimal scalar coupling $-\frac{1}{2} f(h) \mathcal{R}$ to the curvature $\mathcal{R}$ and found a simplified expression for the leading-order gravitational correction to the bounce action

$$
\Delta S_{\text {gravity }} \simeq \frac{\pi^{2}}{6 \bar{M}_{\mathrm{Pl}}^{2}} \int d r r^{5}\left[\frac{h_{0}^{\prime 2}}{2}-V\left(h_{0}\right)-\frac{3}{r} f^{\prime}\left(h_{0}\right) h_{0}^{\prime}\right]^{2} \geq 0
$$

which makes clear that gravity suppresses Minkowski vacuum decay. Going beyond this leading-order approximation we discussed how theories of quantum gravity can affect the result: string models can give a landscape of new vacua, agravity reduces the gravitational correction.

The expansion parameter of thermal corrections is $g / \pi \sim 10^{-1}$ (larger than the expansion parameter $g^{2} /(4 \pi)^{2} \sim 10^{-3}$ of quantum corrections at $\left.T=0\right)$. We found that 2-loop corrections to the thermal potential and one-loop thermal corrections to the Higgs kinetic term change the bounce action by a small amount, at the few \% level, as illustrated in figure 5 . The SM meta-stability boundary in the $\left(M_{t}, M_{h}\right)$ plane gets shifted by $+0.1 \mathrm{GeV}$ in $M_{t}$ by a $3 \%$ increase in the thermal bounce action $S$. Taking into account that the two new effects that we added have opposite sign, figure 4 shows the minor shift in the boundary computed assuming a reheating temperature of $10^{16} \mathrm{GeV}$. Furthermore, we verified that the usual time-independent thermal bounce dominates over time-dependent bounces: this generically happens at large temperatures but was not guaranteed within the SM, given that it is quasi-scale-invariant.

In conclusion, the residual theoretical uncertainty on SM meta-stability bounds is safely smaller than the experimental uncertainty, dominated by the uncertainty on the top mass.

\section{Acknowledgments}

We thank J.R. Espinosa, M. Garny, M. Quiros and V. Rychkov for useful discussions. This work was supported by the grant 669668 - NEO-NAT — ERC-AdG-2014.

Open Access. This article is distributed under the terms of the Creative Commons Attribution License (CC-BY 4.0), which permits any use, distribution and reproduction in any medium, provided the original author(s) and source are credited.

\section{References}

[1] F. Bezrukov, M.Yu. Kalmykov, B.A. Kniehl and M. Shaposhnikov, Higgs boson mass and new physics, JHEP 10 (2012) 140 [arXiv:1205.2893] [INSPIRE]. 
[2] G. Degrassi et al., Higgs mass and vacuum stability in the standard model at NNLO, JHEP 08 (2012) 098 [arXiv: 1205.6497] [INSPIRE].

[3] D. Buttazzo et al., Investigating the near-criticality of the Higgs boson, JHEP 12 (2013) 089 [arXiv:1307.3536] [INSPIRE].

[4] G. Isidori, V.S. Rychkov, A. Strumia and N. Tetradis, Gravitational corrections to standard model vacuum decay, Phys. Rev. D 77 (2008) 025034 [arXiv:0712.0242] [INSPIRE].

[5] J.R. Espinosa, J.-F. Fortin and M. Trépanier, Consistency of scalar potentials from quantum de Sitter space, Phys. Rev. D 93 (2016) 124067 [arXiv: 1508.05343] [INSPIRE].

[6] J.R. Espinosa, Implications of the top (and Higgs) mass for vacuum stability, PoS (TOP2015) 043 [arXiv: 1512.01222] [INSPIRE].

[7] V. Branchina, E. Messina and D. Zappala, Impact of gravity on vacuum stability, arXiv:1601.06963 [INSPIRE].

[8] Y. Goto and K. Okuyama, Numerical analysis of Coleman-de Luccia tunneling, arXiv: 1601.07632 [INSPIRE].

[9] A. Masoumi, S. Paban and E.J. Weinberg, Tunneling from a Minkowski vacuum to an AdS vacuum: a new thin-wall regime, Phys. Rev. D 94 (2016) 025023 [arXiv:1603.07679] [INSPIRE].

[10] A. Rajantie and S. Stopyra, Standard model vacuum decay with gravity, arXiv:1606.00849 [INSPIRE].

[11] O. Czerwińka, Z. Lalak, M. Lewicki and P. Olszewski, The impact of non-minimally coupled gravity on vacuum stability, arXiv:1606.07808 [INSPIRE].

[12] J.R. Espinosa, G.F. Giudice and A. Riotto, Cosmological implications of the Higgs mass measurement, JCAP 05 (2008) 002 [arXiv:0710.2484] [INSPIRE].

[13] A. Kobakhidze and A. Spencer-Smith, Electroweak vacuum (in)stability in an inflationary universe, Phys. Lett. B 722 (2013) 130 [arXiv:1301.2846] [INSPIRE].

[14] A. Salvio, Higgs inflation at NNLO after the boson discovery, Phys. Lett. B 727 (2013) 234 [arXiv: 1308.2244]

[15] M. Fairbairn and R. Hogan, Electroweak vacuum stability in light of BICEP2, Phys. Rev. Lett. 112 (2014) 201801 [arXiv: 1403.6786] [INSPIRE].

[16] A. Hook, J. Kearney, B. Shakya and K.M. Zurek, Probable or improbable universe? correlating electroweak vacuum instability with the scale of inflation, JHEP 01 (2015) 061 [arXiv: 1404.5953] [INSPIRE].

[17] M. Herranen, T. Markkanen, S. Nurmi and A. Rajantie, Spacetime curvature and the Higgs stability during inflation, Phys. Rev. Lett. 113 (2014) 211102 [arXiv:1407.3141] [INSPIRE].

[18] K. Enqvist, T. Meriniemi and S. Nurmi, Higgs dynamics during inflation, JCAP 07 (2014) 025 [arXiv: 1404.3699$]$ [INSPIRE].

[19] A. Kobakhidze and A. Spencer-Smith, The Higgs vacuum is unstable, arXiv:1404.4709 [INSPIRE].

[20] K. Kamada, Inflationary cosmology and the standard model Higgs with a small Hubble induced mass, Phys. Lett. B 742 (2015) 126 [arXiv:1409.5078] [INSPIRE]. 
[21] A. Salvio and A. Mazumdar, Classical and quantum initial conditions for Higgs inflation, Phys. Lett. B 750 (2015) 194.

[22] J.R. Espinosa et al., The cosmological Higgstory of the vacuum instability, JHEP 09 (2015) 174 [arXiv: 1505. 04825] [INSPIRE].

[23] W.E. East, J. Kearney, B. Shakya, H. Yoo and K.M. Zurek, Spacetime dynamics of a Higgs vacuum instability during inflation, submitted to Phys. Rev. D, arXiv:1607.00381 [INSPIRE].

[24] A. Andreassen, D. Farhi, W. Frost and M.D. Schwartz, Precision decay rate calculations in quantum field theory, arXiv: 1604.06090 [INSPIRE].

[25] G.F. Giudice, P. Paradisi and A. Strumia, Indirect determinations of the top quark mass, JHEP 11 (2015) 192 [arXiv:1508.05332] [INSPIRE].

[26] G.W. Anderson, New cosmological constraints on the Higgs boson and top quark masses, Phys. Lett. B 243 (1990) 265 [inSPIRE].

[27] P.B. Arnold and S. Vokos, Instability of hot electroweak theory: bounds on $m_{H}$ and $M_{t}$, Phys. Rev. D 44 (1991) 3620 [inSPIRE].

[28] J.R. Espinosa and M. Quirós, Improved metastability bounds on the standard model Higgs mass, Phys. Lett. B 353 (1995) 257 [hep-ph/9504241] [INSPIRE].

[29] L. Delle Rose, C. Marzo and A. Urbano, On the fate of the standard model at finite temperature, JHEP 05 (2016) 050 [arXiv: 1507.06912] [INSPIRE].

[30] S.R. Coleman and F. De Luccia, Gravitational effects on and of vacuum decay, Phys. Rev. D 21 (1980) 3305 [INSPIRE].

[31] S.R. Coleman, V. Glaser and A. Martin, Action minima among solutions to a class of euclidean scalar field equations, Commun. Math. Phys. 58 (1978) 211 [INSPIRE].

[32] G. Isidori, G. Ridolfi and A. Strumia, On the metastability of the standard model vacuum, Nucl. Phys. B 609 (2001) 387 [hep-ph/0104016] [InSPIRE].

[33] K.S. Stelle, Renormalization of higher derivative quantum gravity, Phys. Rev. D 16 (1977) 953 [INSPIRE].

[34] A. Salvio and A. Strumia, Agravity, JHEP 06 (2014) 080 [arXiv:1403.4226] [INSPIRE].

[35] A. Salvio and A. Strumia, Quantum mechanics of 4-derivative theories, Eur. Phys. J. C 76 (2016) 227 [arXiv: 1512.01237] [INSPIRE].

[36] V. Branchina and E. Messina, Stability, Higgs boson mass and new physics, Phys. Rev. Lett. 111 (2013) 241801 [arXiv:1307.5193] [INSPIRE].

[37] K. Kannike et al., Dynamically induced planck scale and inflation, JHEP 05 (2015) 065 [arXiv: 1502.01334] [INSPIRE].

[38] P.B. Arnold and O. Espinosa, The effective potential and first order phase transitions: beyond leading-order, Phys. Rev. D 47 (1993) 3546 [Erratum ibid. D 50 (1994) 6662] [hep-ph/9212235] [INSPIRE].

[39] Z. Fodor and A. Hebecker, Finite temperature effective potential to order $g^{4}, \lambda^{2}$ and the electroweak phase transition, Nucl. Phys. B 432 (1994) 127 [hep-ph/9403219] [InSPIRE].

[40] I.G. Moss, D.J. Toms and W.A. Wright, The effective action at finite temperature, Phys. Rev. D 46 (1992) 1671 [INSPIRE]. 
[41] D. Bödeker, W. Buchmüller, Z. Fodor and T. Helbig, Aspects of the cosmological electroweak phase transition, Nucl. Phys. B 423 (1994) 171 [hep-ph/9311346] [InSPIRE].

[42] M. Quirós, Field theory at finite temperature and phase transitions, Helv. Phys. Acta 67 (1994) 451 [INSPIRE].

[43] A.D. Linde, Fate of the false vacuum at finite temperature: theory and applications, Phys. Lett. B 100 (1981) 37 [INSPIRE].

[44] J. Garriga, Instantons for vacuum decay at finite temperature in the thin wall limit, Phys. Rev. D 49 (1994) 5497 [hep-th/9401020] [INSPIRE].

[45] A. Ferrera, Bubble nucleation in $\phi^{4}$ models at all temperatures, Phys. Rev. D 52 (1995) 6717 [hep-ph/9510379] [INSPIRE]. 